\title{
Recurrent Haemoptysis Revealing Hughes-Stovin Syndrome
}

\author{
Nedia Moussa ${ }^{1}$, Tasnim Znegui ${ }^{1}$, Mouna Snoussi $^{2}$, Rahma Gargouri $^{1}$, Zouhaier Bahloul ${ }^{2}$, Sourour Abid ${ }^{1}$, Samy Kammoun $^{1}$ \\ ${ }^{1}$ Pneumology Department, Hedi Chaker Hospital, Sfax, Tunisia \\ 2Internal Medicine Department, Hedi Chaker Hospital, Sfax, Tunisia
}

\section{Doi: 10.12890/2021_002810 - European Journal of Case Reports in Internal Medicine - ๑ EFIM 2021}

Received: 31/07/2021

Accepted: $13 / 08 / 2021$

Published: 05/10/2021

How to cite this article: Moussa N, Znegui T, Snoussi M, Gargouri R, Bahloul Z, Abid S, Kammound S. Recurrent haemoptysis revealing huges-stovin syndrome. EJCRIM 2021;8: doi:10.12890/2021_002810.

Conflicts of Interests: The authors declare there are no competing interests.

This article is licensed under a Commons Attribution Non-Commercial 4.0 License

\section{ABSTRACT}

Hughes-Stovin syndrome is a very rare condition with no defined diagnostic criteria. We present the case of a 26-year-old man who had haemoptysis revealing Hughes-Stovin syndrome. We will consider the aetiology, therapeutic and evolutionary aspects of this disease.

\section{LEARNING POINTS}

- Hughes-Stovin syndrome is a very rare disorder of unknown aetiology which can be fatal.

- It is considered a variant of Behcet's disease.

- Early diagnosis and treatment improve prognosis.

\section{KEYWORDS}

Hughes-Stovin syndrome, aneurysms, thrombosis

\section{INTRODUCTION}

Hughes-Stovin syndrome (HSS) is an extremely rare disorder with fewer than 60 cases reported in the literature ${ }^{[1]}$. The disease is characterized by the combination of multiple pulmonary artery aneurysms and peripheral venous thrombosis. The aetiology of this syndrome is still unknown but it is thought to be a variant of Behcet's disease (BD) ${ }^{[2,3]}$.

\section{CASE DESCRIPTION}

A 26-year-old man presented with a recurrent productive cough, haemoptysis and intermittent fever. The chest x-ray revealed a migratory pulmonary basal alveolar opacity. Computed tomography (CT) revealed fluctuating multifocal parenchymal consolidation (Fig. 1). The diagnosis of cryptogenic organizing pneumonia (COP) was made according to the clinico-radiological features and the normal results of investigations eliminating infection and connective tissue disease. The patient received systemic corticosteroid therapy with good clinical outcome but was then lost to follow-up.

Two years later, the patient consulted for massive haemoptysis with respiratory distress related to bilateral pulmonary embolism requiring hospitalization in the intensive care unit, the use of mechanical ventilation and heparin therapy. The aetiological investigation was negative, in particular, immunological tests including antinuclear anti-bodies (ANA) and anti-neutrophil cytoplasmic antibodies (ANCA) were negative, while anticardiolipin, factor $\mathrm{V}$ Leiden mutation gene, factor II prothrombin mutation, homocysteine level, protein C and protein S values were normal. 
After 3 months, the patient was admitted to our department with complaints of recurrent haemoptysis. Physical examination revealed oral and genital ulcers. A complete blood count and biochemical tests were normal. Bronchial endoscopy showed a budding smooth lesion in the middle lobe, which bled on contact. CT pulmonary angiography showed pulmonary embolism of the right lung base with an infra-centimetric lesion of the lower lobe branch of the right pulmonary artery upstream of the thrombosis, prolapsing at the origin of the middle lobe branch, suggesting an aneurysm (Fig.2). Doppler ultrasound of the lower limbs showed evidence of deep vein thrombosis (DVT) in the right leg extending to the sub-renal portion of the inferior vena cava.

The patient was diagnosed with HSS. He was treated with heparin therapy and oral corticosteroid (1 mg/kg/day). He improved significantly during the 7 years of follow-up with no further episodes of haemoptysis reported. He is currently taking $10 \mathrm{mg}$ oral prednisolone each day.

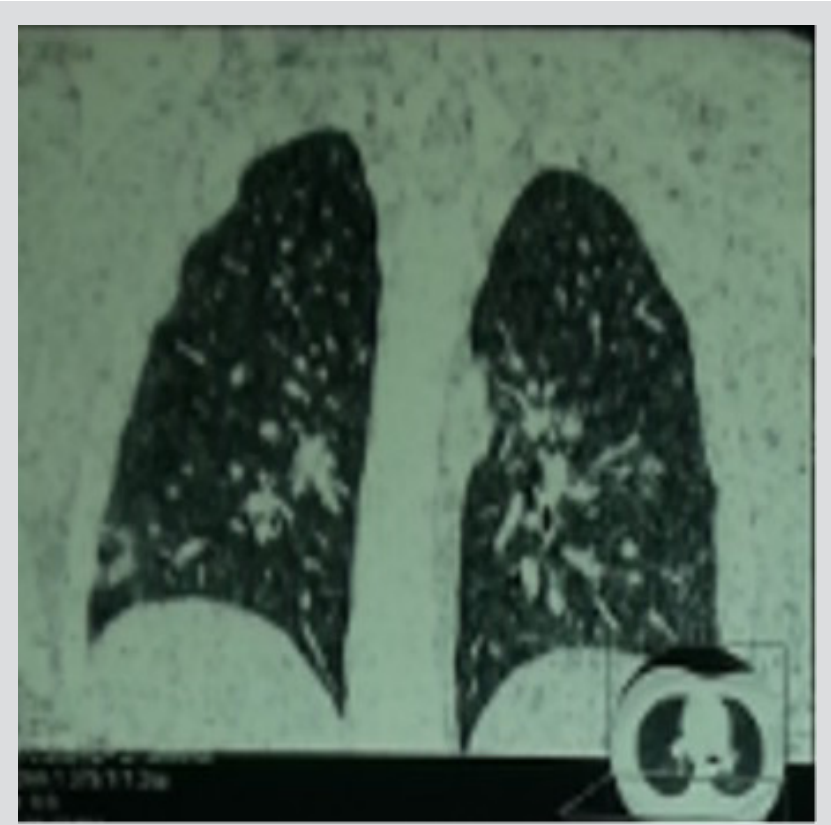

Figure 1. Parenchymal consolidation, reverse halo sign



Figure 2.Computed tomography scan of the chest demonstrating an aneurysm of the lower lobe branch of the right pulmonary artery

\section{DISCUSSION}

Hughes-Stovin syndrome is an extremely rare disease of unknown aetiology. It is characterized by the association of multiple pulmonary and/or bronchial aneurysms and thrombophlebitis. It is considered a variant of BD. This syndrome, first described in 1959,usually affects young men between 12 and 48 years of age ${ }^{[2,3]}$.

The typical presentation of HSS syndrome occurs in three phases: a first stage involving symptoms of thrombophlebitis, a second stage consisting of the formation and enlargement of pulmonary aneurysms, and a third stage of aneurysmal rupture leading to massive haemoptysis and death. The typical presenting features of HSS are related to the first two steps. These signs includecough, haemoptysis, dyspnoea, chest pain, fever, chills, signs of pulmonary hypertension and intracranial hypertension ${ }^{[4]}$.

Aneurysms can be single (as in our patient), multiple, unilateral or bilateral, and usually involve the pulmonary and bronchial arteries resulting in haemoptysis. However, they can occur anywhere in the systemic circulation. Generally, recurrent phlebitis involves the large vessels resulting in thrombus formation. Thrombosis of the vena cava and the right atrium has also been described ${ }^{[5,6]}$.

The aetiology of this syndrome is unknown and its exact pathophysiological mechanism is still uncertain. The pathogenesis of pulmonary artery aneurysms has been attributed to a weakness of the vessel wall due to inflammation ${ }^{[4]}$. Angiodysplasia of bronchial arteries is another debatable hypothesis to account for the vascular changes. Hughes and Stovin's original theory suggested that the aneurysms develop due degenerative changes in the walls of the bronchial arteries, with subsequent changes in the vasa vasorum of the pulmonary artery. This theory was supported by findings of dilated and distorted bronchial arteries and convoluted small branches in digital subtraction angiograms. In addition, the pulmonary artery thrombosis develops in situ due to inflammation of the arterial wall and not as thromboembolism developing from peripheral vein thrombosis ${ }^{[7]}$. 
According to current opinion, HSS is caused by a vasculitis similar to that implicated in BD. Vasculitis in BD results in arterial occlusion, arterial aneurysm, venous occlusion and varices, denoting systemic vessel involvement. It is highly probable that HSS is a cardiovascular manifestation of BD, since in both clinical entities pulmonary aneurysms are characteristic clinical features. In fact, other clinical presentations can overlap significantly; both are more common in young men, the pulmonary manifestations of both can be identical, and the histology of the aneurysms in both entities can be similar ${ }^{[8]}$. In the case of our patient, the HSS revealed the BD. In fact, the diagnosis of $\mathrm{BD}$ is retained according to the international classification criteria (oral and genital ulcers and vascular involvement).

HSS is mostly fatal as a result of massive haemoptysis due to pulmonary/bronchial arterial aneurysm rupture. Likewise, systemic bronchial artery hypertrophy secondary to ischaemia related to the pulmonary artery occlusion could also be the origin of bleeding ${ }^{[5]}$.

There is no standard treatment for HSS. The management of pulmonary vasculitis in HSS should follow the same approach used for its treatment in BD, because, at present, these are the only two conditions known to predispose to pulmonary artery aneurysms with underlying pulmonary arterial vasculitis. The new updated EULAR (European League Against Rheumatism) recommendations for the management of $\mathrm{BD}$ advise the use of high-dose glucocorticoids and cyclophosphamide for the treatment of pulmonary artery aneurysms, while monoclonal anti-TNF antibodies should be considered in refractory cases ${ }^{[9]}$.

In cases of a high risk of major bleeding, embolization should be preferred to open surgery. This procedure is less invasive, enabling selective treatment of multiple bilateral aneurysms ${ }^{[7,10]}$.

Despite the pro-thrombotic tendency and the risk of pulmonary embolism, the need for anticoagulation is not always clear. These patients may still develop thrombosis despite adequate anticoagulation. Anticoagulation should be considered with extreme caution and only in clinical situations where the benefits clearly outweigh the risks ${ }^{[9-11]}$.

In conclusion, HSS has been described as 'incomplete Behçet disease'. It could reveal BD as in our patient. The natural course of this illness is usually fatal. The management of pulmonary vasculitis in HSS should follow the same approach used for its treatment in BD. More studies are required to reach a consensus on the issue of anticoagulation. Early diagnosis and prompt treatment of HSS greatly improve the prognosis.

\section{REFERENCES}

1. Emad Y, Ragab Y, Kechida M,GuffroyA, KindermannM,RobinsonC, etal. A critical analysis of 57 cases of Hughes-Stovin syndrome (HSS). A report by the HSS International Study Group (HSSISG). Int J Cardiol 2021;331:221-229. doi:10.1016/j.ijcard.2021.01.056

2. Weintraub JL, DeMayo R, Haskal ZJ, Susman J. SCVIR Annual Meeting Film Panel Session: diagnosis and discussion of case 1: Hughes-Stovin syndrome. J Vasc Interv Radiol 2001;12(4):531-534. doi:10.1016/S1051-0443(07)61897-1

3. El Aoud S, Frikha F, Snoussi M, Bahloul Z. Moderate hemoptysis caused by Hughes-Stovin syndrome. Clin Pract 2014;4(3):647. doi:10.4081/cp.2014.647

4. Khalid U, Saleem T. Hughes-Stovin syndrome. Orphanet J Rare Dis 2011;6(1):15. doi:10.1186/1750-1172-6-15

5. Abdelbary M, El-Masry A, Rabie MS. Life threatening hemoptysis from Hughes Stovin syndrome: is it that rare? Respir Med Case Rep 2016;19:98-102. doi:10.1016/j. rmcr.2016.08.003

6. Al-Jahdali H. Massive hemoptysis and deep venous thrombosis presenting in a woman with Hughes-Stovin syndrome: a case report. J Med Case Rep 2010;4(1):109. doi:10.1186/1752-1947-4-109

7. Al-Zeedy K, Jayakrishnan B, Rizavi D, Alkaabi J. Hughes-Stovin syndrome and massive hemoptysis: a management challenge. Oman Med J 2015;30(1):59-62. doi:10.5001/ omj.2015.11

8. Chalazonitis AN, Lachanis SB, Mitseas P,ArgyriouP,TzovaraJ,PorfyridesP, et al. Hughes-Stovin syndrome: a case report and review of the literature. Cases J 2009;2(1):98. doi:10.1186/1757-1626-2-98

9. Hatemi G, Christensen R, Bang D,BodaghiB,CelikAF,FortuneF,et al. 2018 Update of the EULAR recommendations for the management of Behçet's syndrome. Ann Rheum Dis 2018;77(6):808-818.doi:10.1136/annrheumdis-2018-213225

10. Emad Y, Ragab Y, El-Marakbi A,SaadA,IbrahimO,Abd-ElhalimA, et al. A case of Hughes-Stovin syndrome (incomplete Behçet's disease) with extensive arterial involvement: unmasking the true face of a rare syndrome. Z Rheumatol 2019:78(4):365-371. doi:10.1007/s00393-019-0618-7

11. Keskin M, Polat G, Ayrancı A, Karadeniz G, Üçsular FD,et al. Insidious Hughes Stovin syndrome: journey from pulmonary embolism to pulmonary arterial aneurysm. Turk Thorac J 2020;21(5):350-353. doi:10.5152/TurkThoracJ.2018.19088 